\title{
THE INFLUENCE OF MAGNETIC MATERIAL DEGRADATION ON THE OPTIMAL DESIGN PARAMETERS OF ELECTROMAGNETIC DEVICES
}

\author{
Ahmed Abdallh and Luc Dupré \\ Department of Electrical Energy, Systems \& Automation, Ghent University, Belgium
}

Electromagnetic devices (EMDs) are normally designed based on the properties of the used magnetic material provided by the electrical steel manufacturers. However, it is well-known that the characteristics of magnetic materials inside EMDs are altered during the production of these devices. Mechanical stresses due to electrical steel cutting, clamping and shrink fitting cause a dramatic deterioration of the magnetic material properties [1]. Consequently, the designed EMD based on the original magnetic material may not achieve the targeted performances [2].

In this paper, we investigate the effect of magnetic material degradation on the optimal design parameters of EMDs. First, in order to take the effect of magnetic material degradation into account, a coupled magneto-mechanical finite element based model is solved by incorporating the mechanical stress distribution into the constitutive laws of the magnetic material of the EMD, i.e. single-valued $B-H$ curve and loss parameters. Based on the mechanical stress distribution, an optimization problem is formulated to modify the design strategy of the studied EMD in order to reach specific performances.

Specifically, in this paper, a surface mounted permanent magnet synchronous machine (PMSM) is redesigned by changing some design parameters, i.e. stack length, number and cross section of the excitation windings, for compensating the effect of the magnetic material degradation in order to reach predefined particular performances, i.e. efficiency. To this end, an optimization problem is solved by minimizing iteratively the following objective function:

$$
\tilde{\mathbf{x}}=\underset{\mathbf{x}}{\arg \min } O F(\mathbf{x}), \quad O F(\mathbf{x})=\left\|\eta_{s}(\mathbf{x}, \mathbf{u}(\sigma))-\eta_{t}\right\|^{2}
$$

with $\mathbf{x}$ being the vector containing the design parameters, i.e. $\mathbf{x}=[l, n, s]$, where $l, n$, and $s$ are the stack length, number and cross section of the excitation windings, respectively. $\eta_{t}$ is the target efficiency of the motor. $\eta_{s}$ is the corresponding simulated quantity using the material parameters $\mathbf{u}$, i.e. single-valued $B-H$ curve and loss parameters, affected by the distribution of the mechanical stress $\sigma$ in the magnetic core material.

The results of the optimization problem show that the PMSM can be redesigned to achieve the target efficiency; however, the overall cost of the machine is increased by almost $10 \%$. The presented study can help the electrical machine designer for a better machine design.

[1] TAKAHASHI, N. et al.: Journal of Magnetism and Magnetic Materials, 320 (2008), E925-E928.

[2] ABDALLH, A. - DUPRÉ, L.: book chapter in Advanced Magnetic Materials, InTech, Croatia, ISBN 978-953-51- 0637-1, 2012.

Name and e-mail of corresponding author:

Dr.Ahmed Abdallh, Ahmed.Abdallh@UGent.be 